\title{
SOBRE LAS VENTAJAS DE LA ALIANZA DEL PACÍFICO PARA COLOMBIA
}

\author{
Iván Alejandro Trujillo Acosta*
}

Fecha de recibido: Enero 10 de 2014.

Fecha de aprobado: Junio 3 de 2014.

Artículo de Reflexión

Forma de citación: Trujillo, I. A. (2014). Sobre las ventajas de la Alianza del Pacífico para Colombia. Revista Prolegómenos, Derechos y Valores, 17, 33, 159-172.

\section{Resumen}

La Alianza del Pacífico (AP) es un área de integración de interés en las relaciones internacionales. No obstante, existe un vacío en la literatura sobre las ventajas de ésta para la política exterior de Colombia. Este artículo busca analizar las ventajas de la AP para Colombia. Se defiende la idea que la AP ofrece ventajas para la inserción internacional de Colombia basadas tanto en las características de esa área de integración como en las oportunidades que ofrece para su política exterior. La AP es un área de integración que tiene en sus instituciones una agenda definida de integración, la cosecha temprana de las negociaciones, el seguimiento a las cumbres y una presidencia pro témpore eficaz. Por otra parte, le ofrece a Colombia un área de integración sin tensiones con otros actores, instrumentos para diversificar sus relaciones con el mundo y una opción de liderazgo regional desde la persuasión.

\section{Palabras clave}

Integración, Alianza del Pacífico (AP), Colombia, Nuevo Multilateralismo, Política Exterior.

\section{THE ADVANTAGES OF PACIFIC ALLIANCE TO COLOMBIA}

\begin{abstract}
The Pacific Alliance (PA) is an integration area of interest in the international relations. Nevertheless, there is a lack in the literature on the advantages that it offers to Colombia's foreign policy. This article analyzes the advantages of PA for Colombia. It defends that the PA offers advantages to Colombia based on its characteristics and the opportunities that it offers for its Foreign Policy. On the one hand, The PA has within its institutions a defined agenda, follow-up on the high level meetings and an effective protempore presidency. On the other hand, it offers to Colombia the opportunity to build
\end{abstract}

* Politólogo de la Universidad Nacional, Especialista en Gerencia Pública y Control Fiscal de la Universidad del Rosario y candidato a Magister en Análisis de Problemas Políticos, Económicos e Internacionales Contemporáneos de la Universidad Externado de Colombia. Actualmente trabaja en el Ministerio de Relaciones Exteriores de Colombia como funcionario de la Carrera Diplomática y Consular. 
an integration area without issues with other actors, instruments to diversify its foreign relations and a regional leadership option based on persuasion.

\title{
Key words:
}

Integration, Pacific Alliance (PA), Colombia, New Multilateralism, Foreign Policy.

\section{AS VANTAGENS DO PACÍFICO ALIANÇA PARA A COLÔMBIA}

\begin{abstract}
Resumo
A Aliança do Pacífico (PA) é uma área de integração de interesses nas relações internacionais. No entanto, há uma carência na literatura sobre as vantagens que ele oferece para a política externa da Colômbia. Este artigo analisa as vantagens do PA para a Colômbia. Ele defende que a PA oferece vantagens para a Colômbia com base em suas características e as oportunidades que ela oferece para a sua política externa. Por um lado, a PA tem dentro de suas instituições uma agenda definida, acompanhamento nas reuniões de alto nível e uma presidência pro-tempore eficaz. Por outro lado, oferece a Colômbia a oportunidade de construir uma área de integração sem problemas com outros atores, instrumentos para diversificar suas relações externas e uma opção de liderança regional com base na persuasão.
\end{abstract}

\section{Palavras-chave}

Integração, Pacific Alliance, Colômbia, Novo multilateralismo, Política externa.

\section{INTRODUCCIÓN}

La Alianza del Pacífico (AP) es un área de integración conformada por Colombia, Perú, Chile y México (Declaración de Lima, 2011)1, para promover la integración con proyección hacia Asia Pacífico (Declaración de Lima, 2011 y Acuerdo Marco de la Alianza del Pacífico, 2012). Ha tenido la atención de la prensa, los centros de pensamiento y diversos autores, los cuales destacan su importancia, sus implicaciones geopolíticas y sus éxitos

1 No obstante, vale la pena aclarar que Panamá fue observador de la Alianza del Pacífico desde su creación. Para el momento de la realización de este artículo Costa Rica avanza en su proceso de adhesión a la AP como un nuevo país miembro de esa Área de Integración. Cfr. Alianza del Pacífico. Declaración sobre el proceso de adhesión de Costa Rica a la Alianza del Pacífico. Cartagena, 10 de febrero de 2014. en materia de comercio e integración. Unos actores presentan a la Alianza como un bloque comercial tan grande como una potencia económica mundial (Dade, Carlo \& Meecham, 12013 ); otros, como una iniciativa de países con visiones comunes en competencia con otros bloques de integración (Farnsworth, 2013 \& Malamud, 2013) y otro grupo como un área de integración prometedora para el comercio y la inversión (Samuel, 2014 \& Ramírez, 2013).

Buena parte de los estudios sobre la AP la analizan como un actor unitario en interacción con potencias globales y/o regionales (Dade \& Meecham, 2013, Ramírez, 2013 \& Farnsworth, 2013). En contraste, una escasa minoría presenta el papel de los países dentro de la Alianza; aunque más como parte de una argumentación sobre la uniformidad de visiones o de políticas dentro de ese espacio de integración (George, 2014, p. 6-25 \& San Francisco, 2013). En la 
literatura sobre el tema no se analizan en detalle las ventajas de la Alianza para los Estados participantes como Colombia, más allá del acercamiento al Asia Pacífico.

Así, si bien hay un consenso aparente en que la AP representa un logro para la política exterior de sus países miembros, la literatura existente no analiza en detalle las ventajas que le ofrece la Alianza a Colombia. Tampoco detalla las oportunidades que brinda la inserción en esa área de integración al país. Así, existe la necesidad de identificar las ventajas y beneficios de la participación de Colombia en ese espacio de integración. Surge la problemática de analizar cuáles son las ventajas de la AP para Colombia en su política exterior.

En ese contexto, este artículo sostiene que la AP significa ventajas para la inserción internacional de Colombia que se basan tanto en las características de esa área de integración como en las oportunidades que ofrece para su política exterior. En ese sentido, a continuación se presentarán las ventajas que ofrece la AP desde sus características, seguidas de las oportunidades que ofrece para la política exterior del país.

Esta reflexión se desarrolla con un enfoque ecléctico, que emplea elementos de los estudios sobre la integración regional y del análisis de política exterior. En ese sentido, el nuevo multilateralismo y la política exterior de Colombia son los puntos de partida de este aporte a la discusión.

La discusión sobre el nuevo multilateralismo tiene varias visiones. Caporaso define al multilateralismo como un principio de organización en el cual los Estados construyen instituciones indivisibles, con principios generales de conducta y difusa reciprocidad (1992, p. 601,602). Por otra parte, Ledger caracteriza al multilateralismo como un arreglo institucional de tres o más Estados con la participación de otros actores, así como diferencia un viejo y nuevo multilateralismo, en el cual el nuevo multilateralismo se distingue por ser Estado céntrico, pro témpore, enfocado hacia la concertación política sobre la integración, defensivo hacia los Estados Unidos e intergubernamental (2010, p. 1-3).

En contraste, Ardila expone las siguientes características del nuevo multilateralismo en América Latina: es presidencialista pero buscando una mayor institucionalización de la toma de decisiones, es pro témpore, evitando burocracias supranacionales, busca una proyección externa hacia el liderazgo regional, se basa en valores identitarios latinoamericanos, es cooperativo y autónomo de Estados Unidos, es flexible en sus organizaciones y se enmarca en nuevos liderazgos regionales (2012, p. 3-5). Esta mirada ofrece elementos importantes para el estudio de la integración regional desde el nuevo multilateralismo en América Latina que, debido a su amplitud y profundidad, se tiene en cuenta en este artículo sin excluir los aportes de otros autores.

Los estudios de política exterior (Ardila, Cardona, \& Ramírez, 2005; Cardona, 2011; Borda \& Tickner, 2011), ${ }^{2}$ caracterizan a la política exterior de Colombia en la actualidad como una política de reencuentro regional (Ramírez, 2011, p. 80-84) o de transición en su inserción internacional (Ardila, 2011, p.115-118). Eso significa que las prioridades de la política tuvieron un cambio con la administración actual, de una política en función de la seguridad con socios excluyentes (Ardila, 2011, p. 101) a una política de promoción de las relaciones regionales, comercio, cooperación y diversificación regional (Ramírez, 2011, p. 95).

En ese contexto, las prioridades de la política exterior de Colombia comprenden el fortalecimiento de las relaciones con América Latina y el Caribe, la diversificación de las relaciones con socios tradicionales y la inserción de Colombia en Asia Pacífico, entre otras líneas de acción ${ }^{3}$.

2 Los estudios sobre la política exterior de Colombia han tenido un desarrollo en el país en el marco de las relaciones internacionales.

3 Al respecto, véanse las prioridades de la política internacional de Colombia 2010-2014 en DNP (2010, pp. 519-523). 
Así mismo, los mecanismos para su ejecución comprenden la diplomacia, los instrumentos de diálogo y cooperación tanto bilaterales como regionales, los acuerdos de comercio y las áreas de Integración, entre otros, desarrollados de manera más amplia por Ardila (2011, p. 104, 113).

\section{Las ventajas de la AP desde sus características institucionales}

La AP tiene características ventajosas para Colombia en su agenda, resultados y funcionamiento en el marco del nuevo multilateralismo. $\mathrm{Al}$ respecto, la AP tiene una agenda definida de integración, tiene sólidos resultados, previene la «cumbritis» y no promueve la burocracia en su institucionalidad. Estas características se examinarán a continuación.

\section{Una agenda de integración definida}

La AP tiene una agenda definida de integración en sus instituciones. Al respecto, el Acuerdo Marco de la AP dirige el trabajo de sus países miembros hacia la integración, el crecimiento económico y la proyección al Asia Pacífico. El Acuerdo define como objetivos de la Alianza construir un área de integración profunda hacia la libre circulación de bienes, capitales, servicios y personas; impulsar mayor crecimiento, desarrollo y competitividad de las economías, así como constituirse en una plataforma de articulación política, integración y proyección hacia el Asia Pacífico, según lo estipula el Acuerdo Marco en su artículo 3.

Asimismo, se basa en valores comunes de integración y democracia, que se expresan en el compromiso con la democracia como sistema político, y en la economía de mercado. En primer lugar, la Declaración de Lima parte de ratificar el compromiso de privilegiar la integración como un instrumento efectivo para el desarrollo, la promoción del comercio y la competitividad de los países hacia el Asia Pacífico $^{4}$. En segundo lugar, la AP estableció

4 Alianza del Pacífico. Declaración de Lima. como requisitos para sus países miembros el respeto a la democracia, el Estado de Derecho y los Derechos Humanos (Declaración de Lima, 2011).

En tercer lugar, según San Francisco (2013), la Alianza es un conjunto de ideas y principios comunes de integración sobre bases de democracia y libre mercado:

La Alianza del Pacífico es una unión de ideas y principios, sin estructuras costosas o excesiva burocracia. Se podría decir que detrás de ella subyace el valor de la libertad. Es interesante comprobar que esto se da en países que tienen gobiernos de tendencias o corrientes políticas distintas y con historias también diferentes, pero que han abrazado dos fórmulas institucionales para su desarrollo social: la democracia como sistema político, y la economía de mercado en el ámbito económico. (p. 7)

La AP define su agenda de integración como una serie de acciones para el alcance de los objetivos de la alianza que incluyen, entre otras, liberalizar el intercambio comercial de bienes y servicios, avanzar hacia la libre circulación de capitales y la promoción de inversiones, facilitar el comercio entre los países miembros, facilitar el movimiento de personas al interior de la Alianza, prevenir y contener la delincuencia organizada transnacional, y promover la cooperación entre los países (Acuerdo Marco, artículo 2). De esta manera, existe una agenda específica de integración que previene la discusión de temas de coyuntura que desvíen la agenda hacia la concertación.

Según Ledger, una de las características del nuevo multilateralismo es la prevalencia de la concertación política sobre la integración (2010, p. 3). Esa característica señala una de las principales fallas de los grupos regionales. En ese sentido, Serbín señala que una de las debilidades de la Unión de Naciones Suramericanas (UNASUR) consiste en sus limitaciones para hacer frente a las tensiones entre sus miembros (2009, p. 154-156). Así 
mismo, según Rojas, una de las limitaciones de la Comunidad de Estados Latinoamericanos y Caribeños (CELAC) es su baja capacidad para construir un mecanismo de toma de decisiones que genere consenso en los temas controversiales y las crisis en medio de un ambiente de mutua desconfianza en la región (2010, p. 7).

Como prueba de ello, la Declaración de Cartagena impartió los siguientes mandatos concretos en la agenda de integración a los ministros de relaciones y a los ministros de comercio de sus países miembros para el primer semestre de 2014, entre otras iniciativas:

- Con el fin de profundizar el libre movimiento de bienes, se realizará un análisis de productos agropecuarios para el comercio, concluir el capítulo de mejora regulatoria y acordar acuerdos de cooperación regulatoria en el subsector cosméticos;

- Se acordó continuar la negociación en materia de transporte aéreo y otros servicios, con el fin de impulsar el libre movimiento de servicios;

- En materia de libre movimiento de capitales, se acordó en compartir experiencias en torno a posibilidades de integración en mercados emergentes, concluir el mecanismo de intercambio de información fiscal entre los países, compartir información tributaria sobre transacciones internacionales y evaluar un futuro Fondo de Desarrollo de Infraestructura para la AP;

- Así mismo, se dieron las instrucciones de concluir un instrumento para facilitar el trabajo de los jóvenes durante sus vacaciones y fortalecer la Plataforma de Intercambio de Información Inmediata para la Seguridad Migratoria de la Alianza con el fin de promover el libre tránsito de personas.

Mandatos que se reflejaron en los siguientes resultados de la Declaración de Punta Mita (2014):

- En el comercio de bienes, se identificaron productos agropecuarios con potencial exportador, se estableció un mecanismo de intercambio de información sobre insumos agrícolas y se registraron avances en la puesta en funcionamiento de un acuerdo de cooperación entre las autoridades sanitarias de los países miembros de la AP;

- En los temas de capitales, se registraron avances en la discusiones sobre el «Fondo para Desarrollo de Infraestructura de la Alianza del Pacífico»;

- En movilidad de personas, se fortaleció la «Plataforma de Intercambio de Información Migratoria de la Alianza del Pacífico», así como se firmó un acuerdo para implementar un programa de vacaciones y trabajo para los jóvenes de los cuatro países.

Y a su vez, esas iniciativas de integración se concretaron en los siguientes mandatos relacionados en la Cumbre de junio de 2014 (Declaración de Punta Mita, p. 7-9), entre otros:

- En materia de comercio de bienes, se instruyó a continuar el trabajo para potenciar el comercio intra Alianza e iniciar labores para potenciar las exportaciones hacia terceras economías, así como formalizar los acuerdos de cooperación regulatoria acordados entre las autoridades de los países para impulsar el subsector cosméticos;

- Para el comercio de servicios, se continuarán los desarrollos en materia de telecomunicaciones y comercio electrónico;

- En los temas de capitales, se acordó elaborar un estudio para identificar barreras y áreas de oportunidad de las inversiones, así como el trabajo coordinado en proyectos para contribuir a un ambiente de mayor certidumbre para los inversionistas;

- En movilidad de personas, se acordó continuar con los intercambios de información, las actividades de trabajo y avanzar en los acuerdos legales que contribuyan a la plena movilidad de personas.

De esta manera, los compromisos y mandatos mencionados constituyen pruebas, sin lugar a dudas, de la agenda coherente y precisa de integración de la Alianza en el futuro inmediato. 


\section{Un área de integración con resultados}

La AP ha mostrado resultados múltiples a corto plazo, tales como la puesta en funcionamiento del Mercado Integrado Latinoamericano $(\mathrm{MILA})^{5}$, la eliminación de visas de viajero para sus países miembros y la continuidad del proceso integración con cambios de Gobierno en sus países miembros, entre otros (Dade y Meechan, 2013, p. 7-8). Este éxito se basa en el método de cosecha temprana de los acuerdos negociados, el cual permite la construcción pragmática de compromisos consecutivos de integración a partir de la negociación de cada asunto por separado al interior de la Alianza (Dade \& Meechan, 2013).

Según Dade y Meechan (2013):

(...) The Pacific Alliance countries have adopted a "harvest" approach to forging agreements. In short, the approach allows for coming to agreements piecemeal, rather than once all issues have been agreed upon. The AP is above all else pragmatic - not, as with many similar groups, an ideological or deeply strategic undertaking. It was created to resolve specific issues that hinder the countries' abilities to both position themselves with respect to trade and negotiate with Asia through collective action (p. 2).

Adicionalmente, la AP parte de la base de los tratados de libre comercio entre sus miembros (Declaración de Lima, 2011) para construir la integración, hecho que garantiza un com-

5 El MILA es un mercado integrado de capitales conformado, inicialmente, por las bolsas de valores de Colombia, México, Chile y Perú. Este mercado permite la realización de transacciones bursátiles en tiempo real mediante una plataforma de comercio electrónico entre los cuatro países, constituyendo un mercado común dentro de esta área de integración. (Dade \& Meecham 2013, p. 7). Actualmente, se aceptó la Bolsa Mexicana de Valores (BMV) al MILA, se aprobó el modelo operativo y los sistemas que harán efectiva su conexión. ( Alianza del Pacífico. Declaración de Punta Mita, p. 3). promiso duradero de sus países miembros desde su vinculación ${ }^{6}$.

El MILA es un ejemplo exitoso del método de cosecha temprana de acuerdos negociados. La primera fase del MILA, que se completó en 2011, construyó los cimientos del proyecto, implementando un sistema de comunicaciones las transacciones bursátiles y asegurando los derechos de publicidad y de solicitud de transacciones en los países participantes. La segunda fase del MILA, actualmente en curso, trabaja en reducir ineficiencias de la primera fase, reduciendo la intermediación en el mercado y los costos de transacción entre los países. Así, este mercado integrado atestigua la eficacia del método mencionado, al probar que las partes pueden sobreponerse a los obstáculos, negociando progresivamente acuerdos que construyen confianza para sobrellevar los temas más sensibles (George, 2014, p. 29).

Finalmente, la AP logró en la Cumbre de Cartagena de febrero de 2014 la firma de un Protocolo Adicional al Acuerdo Marco para la integración profunda y comprensiva entre sus países miembros. Según la Declaración de Cartagena, «(...) constituye el instrumento central para la liberalización de bienes, servicios e inversiones» frente al cual se resalta la decisión de eliminar el 92 por ciento de los aranceles con su entrada en vigor y eliminar el 8 por ciento restante de forma gradual (Declaración de Cartagena, 2014). De igual manera, según esta Declaración, se espera que «(...) proveerá estabilidad y seguridad jurídica en materia comercial (...)» para una mayor integración de las cadenas regionales de valor que harán compe-

6 Los Vicecancilleres y Viceministros de Comercio acordaron en la IX reunión del Grupo de Alto Nivel (GAN) de 2012 que los Estados solo pueden convertirse en candidatos a adherir a la AP cuando tengan Tratados de Comercio con al menos dos miembros de la Alianza. (Alianza del Pacífico. Lineamientos sobre la participación de Estados Observadores de la Alianza del Pacífico. Numeral 6). Al respecto, esa medida garantiza un grado de integración mínimo para la vinculación a la Alianza. 
titiva la región. Así, la AP logra consolidar una Zona de Libre Comercio como consecuencia de su integración (Protocolo Adicional al Acuerdo Marco de la Alianza del Pacífico, art. 1.1)

\section{Un área sin cumbritis ni burocracia}

La AP, a diferencia de otros grupos regionales, no padece de «cumbritis». Esta expresión hace referencia al problema de la excesiva realización de reuniones de jefes de Estado y Gobierno sin resultados concretos. Al respecto, Carlos Portales (2012), en un artículo sobre las cumbres de la CELAC, UNASUR y Mercosur, afirma que las múltiples cumbres se caracterizan por un dialogo desarticulado sin mayores resultados como conjunto. Asimismo, establece que las cumbres por si mismas no garantizan el éxito de esos foros multilaterales: los diálogos de mandatarios, si bien permiten desactivar crisis inmediatas, tienen limitaciones para la coordinación de las políticas sin el dialogo transnacional de sus ministros clave de gobierno.

Esto se explica en el seguimiento a las reuniones de presidentes dentro de la Alianza. La realización de reuniones de seguimiento a la integración por parte de los ministros de relaciones exteriores y de comercio $^{7}$, del Grupo de Alto Nivel ${ }^{8}$ y de los grupos de trabajo9, garantiza un trabajo fluido hacia la integración antes de cada reunión de presidentes, donde solo se avalan los avances logrados y se proponen nuevas metas de integración con un alcance definido. Al respecto, Ramirez (2013)

7 Según el Acuerdo Marco, las reuniones de ministros de relaciones exteriores y de comercio se realizan una vez al año, con la coordinación de la Secretaria protémpore de cada país. (Alianza del Pacífico. Acuerdo Marco... Óp. Cit. Artículos 4 y 7).

8 El Grupo de Alto Nivel es convocado por la reunión de ministros de relaciones y de comercio, cuando este lo considere necesario. (Alianza del Pacífico. Acuerdo Marco... Óp., Cit. Art. 4).

9 Los Grupos de Trabajo son definidos por la reunión de ministros para la consecución de los objetivos y la realización de las acciones de la AP. (Alianza del Pacífico. Acuerdo Marco... Óp., Cit. art. 4). indica que: "In its first two years, the Pacific Alliance has convened six presidential summits. In notable contrast with the gatherings of other Latin American groups, their communiqués provided specific statements and detailed timelines to advance agreed-upon goals».

La AP no promueve la burocracia en su institucionalidad, debido al trabajo de la Presidencia pro témpore de cada país. Al respecto, cada año un país miembro de la Alianza coordina el proceso de integración Acuerdo Marco, artículo 7), apoyando las actividades de los grupos de trabajo $^{10}$. Esta característica de la Alianza es acorde con el nuevo multilateralismo, el cual, según Ardila (2012) tiene como rasgo su carácter pro témpore, lo que evita burocracias supranacionales. En ese sentido, se busca construir una institucionalidad común a partir del trabajo de los países sin generar una burocracia internacional para la integración.

De esta manera, la agenda definida de la AP, sus éxitos basados en la cosecha temprana de las negociaciones, el seguimiento a las cumbres y su eficaz presidencia pro témpore hacen de la AP un área de integración atractiva por la credibilidad de sus instituciones en el marco del nuevo multilateralismo.

\section{Las ventajas de la AP desde las oportunidades que ofrece}

La AP ofrece varias ventajas a la política exterior de Colombia. Le facilita profundizar sus relaciones regionales sin generar tensiones con terceros países ni otros grupos regionales. Asimismo, diversifica sus relaciones exteriores con otras regiones y países. También potencia nuevos temas de relacionamiento desde la cooperación internacional. Finalmente, viabiliza un liderazgo para el país mediante la construcción de consensos.

10 Es importante tener en cuenta que según el Acuerdo Marco los grupos de trabajo tienen como finalidad la consecución de los objetivos y la realización de las acciones de la AP (artículo 4). 


\section{Un área de integración abierta al mundo}

La AP no genera tensiones con terceros países ni con otros grupos regionales, porque es un área de integración abierta a la participación de otros países. Al respecto, está abierta a la participación de todos los países que compartan los mismos valores y compromiso con la integración ${ }^{11}$. Prueba de ello es la participación como países observadores de Canadá, Australia y España (Declaración de Cadiz), (todas potencias medias globales ${ }^{12}$ ); así como la participación de China y Estados Unidos. Con la aceptación de Turquía y Corea del Sur la Alianza del Pacífico suma 20 Países Observadores.

Así, la AP no excluye a ningún país en sus instituciones ni compite con otros países por su proyecto de integración. Según Serbín (2009) no sucede lo mismo en otros casos de integración regional, como Brasil y Venezuela, que compiten en América del Sur por el liderazgo de sus visiones de política exterior a través del ALBA y el Mercosur (p. 146-150).

En ese sentido, la AP no es excluyente con otros procesos de integración. No excluye ni elimina el trabajo de otros grupos regionales ${ }^{13}$ y está

11 Los lineamientos sobre Estados observadores de la Alianza del Pacífico dicen que cada «(...) Estado Observador deberá compartir los principios y objetivos establecidos en el Acuerdo Marco de la Alianza del Pacífico» numeral 2.

12 Desde hace varios años Palou (1993) caracterizaba a esos países como potencias medias del mundo, entendidas como «(...) Estados que, debido a su dimensión (territorial, demográfica, económica, político-diplomática o militar) o a su situación geopolítica en una región determinada, presentan la capacidad y la voluntad necesarias para ejercer una cierta influencia en determinadas áreas de las relaciones internacionales» (p. 15). Sobre la identificación de España, Canadá y Australia como potencias medias, entre otros países (Palou, 1993, p. 18-19).

13 El Acuerdo Marco de la Alianza del Pacífico establece que «Las decisiones del Consejo de Ministros y otros acuerdos adoptados en el ámbito de la Alianza del Pacífico no reemplazarán ni modificaran los acuerdos económicos, comerciales y de integración bilaterales, regionales o multilaterales vigentes entre las partes» (Artículo 8). abierta a la observación y colaboración con ellos (Declaración de Lima). Adicionalmente, esta abierta al diálogo informativo con el Mercosur y a la realización de actividades académicas con el Mercosur y con otras partes de la región, incluyendo Centroamérica y el Caribe (Declaración de Punta Mita). De esta manera, la AP abre un espacio valioso a Colombia para profundizar su integración con los países de la Alianza, facilitando su comercio y potenciando su competitividad.

\section{Un espacio para diversificar relaciones exteriores}

La AP le permite diversificar sus relaciones exteriores en términos geográficos y temáticos a partir de las acciones conjuntas en el marco de la AP. En ese sentido, la apertura de misiones diplomáticas y comerciales conjuntas con los países miembros de la Alianza en Ghana, Turquía, Argelia, Marruecos y Vietnam, Azerbaiyán y Singapur es una acción efectiva para diversificar la política exterior con otros países.

Así mismo, la AP logra acercar a sus países miembros con los países de Asia Pacífico. La incorporación de China, Japón, Corea del Sur, Australia, Nueva Zelandia y Singapur como países observadores de la Alianza (Declaración de Cartagena, 2014), prueba la efectividad de la AP para fortalecer las relaciones con Asia Pacífico. La inclusión de esos Estados como miembros observadores no solo significa el interés de estos en participar en la Alianza, también significa la posibilidad de potenciar las relaciones existentes con los países de Asia Pacífico hacia un área de integración y cooperación.

De igual manera, la AP trabaja a nivel multilateral con el Banco Interamericano de Desarrollo (BID) y la Organización para la Cooperación y Desarrollo Económicos (OCDE), en beneficio de objetivos comunes. La AP recibe el apoyo y la asistencia técnica del BID para las diferentes dimensiones de trabajo de su proceso de integración (Declaración de Punta Mita, 2014). Por otra parte, los países de la Alianza 
comparten una Misión de representación ante la OCDE en París e iniciaron trabajos para facilitar la adopción de políticas comunes de impulso a la competitividad e internacionalización de las PYMES, entre otras actividades (Declaración de Punta Mita, 2014).

\section{Una oportunidad para potenciar nuevos temas en la agenda}

La AP promueve nuevos temas en las relaciones de Colombia con sus países miembros y observadores. Al respecto, la Plataforma de Cooperación del Pacífico abre nuevas posibilidades de relacionamiento con esos países, a partir de temas de la agenda regional como la cooperación en educación, investigación sobre cambio climáti$\mathrm{co}^{14}$ y promoción de las pymes ${ }^{15}$, potenciándolos a través de iniciativas de cooperación.

La plataforma de cooperación de la AP, establecida mediante el Memorando de Entendimiento suscrito por los cancilleres de los países miembros en 2011, es un espacio para la cooperación que construyeron los países en el marco de la $\mathrm{AP}^{16}$. La Plataforma permite a los países miembros la cooperación bajo diversas modalidades, tales como la realización de iniciativas, los estudios conjuntos, los intercambios de información, los intercambios de experiencias, la asistencia técnica y la conformación de redes, entre otras ${ }^{17}$. El financiamiento de

14 La red científica de la Alianza del Pacífico provee un espacio el intercambio de conocimientos y trabajo colaborativo para la investigación en cambio climático entre los académicos miembros de la red. (Alianza del Pacífico, 2013)

15 Alianza del Pacífico. Memorando de Entendimiento entre el Gobierno de los Estados Unidos Mexicanos, el Gobierno de la República de Colombia, el Gobierno de la República de Chile y el Gobierno de la República del Perú sobre la plataforma de cooperación del Pacífico. Al respecto, tanto Dade y Mecham (2013) como Ramírez (2013) hacen menciones favorables sobre los avances en ese sentido.

16 Alianza del Pacífico. Memorando de Entendimiento (artículo 1)

17 Alianza del Pacífico. Memorando de Entendimiento las actividades se realiza aportes de recursos específicos de los países bajo la modalidad de costos compartidos o con el apoyo complementario de diversos actores ${ }^{18}$. Los sectores en que se han desarrollado actividades dentro de la plataforma han sido ciencia y tecnología, innovación, medio ambiente y cambio climático, promoción de las pymes y educación ${ }^{19}$.

En materia de Ciencia y Tecnología, los países miembros de la AP han realizado trabajos exploratorios para la conformación de una red de investigación en el tema de cambio climático ${ }^{20}$. Al respecto, se realizó el primer Foro Oportunidades de Colaboración en Investigación sobre Cambio climático en los Países de la Alianza del Pacifico en Santiago Chile en 2013, con la participación de 32 representantes de los gobiernos, investigadores y científicos de los países miembros ${ }^{21}$. En el encuentro, se discutieron el estado del arte del cambio climático en los países miembros, la identificación de intereses de investigación, aplicación y líneas de integración comunes así como la identificación de posibles proyectos comunes ${ }^{22}$, en las áreas de mitigación, adaptación y ciencia del cambio frente a ese fenómeno ${ }^{23}$.

Adicionalmente, se han realizado reuniones de puntos focales, se cuenta con un comité científico y se está construyendo un reglamento para la coordinación científica de la red ${ }^{24}$. Se espera

(artículo 3)

18 Alianza del Pacífico. Memorando de Entendimiento (artículo 5)

19 Alianza del Pacífico. Memorando de Entendimiento (artículo 2)

20 Alianza del Pacífico (2014) Proyecto Red de Investigación Científica en materia de Cambio Climático.

21 Alianza del Pacífico (2013) Científicos de la Alianza del Pacífico debatieron en Chile sobre cambio climático.

22 SELA (2013) Científicos de países de la Alianza del Pacifico debaten en Chile sobre cambio climático.

23 SELA (2013) Ibíd.

24 Alianza del Pacífico (2014) Proyecto Red de Investigación Científica en materia de Cambio Climático. 
que con estos acercamientos se logre consolidar una red de investigación en materia de cambio climático para la construcción de conocimiento especializado frente a ese fenómeno.

En materia de promoción de las Pequeñas y Medianas Empresas (PYMES), la AP logró consolidar varios avances. La AP conformó un Grupo Técnico de PYMES en la Cumbre de Cartagena de 2014 (Declaración de Cartagena) y el Grupo presentó una agenda de emprendimiento para la PYMES en la Cumbre de Punta Mita de junio de 2014. La agenda incluye un mecanismo de financiamiento, acompañamiento e inversión para los emprendedores de los cuatro países, así como una red de apoyo para los emprendedores y el fomento de la cultura emprendedora en los cuatro países (Declaración de Punta Mita). En ese sentido, la Cumbre instruyó el mandato presidencial de explorar con el BID y el Fondo Multilateral de Inversiones (FOMIN) la creación del mecanismo mencionado (anexo Declaración de Punta Mita, 2014).

Por otra parte, la AP realizó su primer Foro regional sobre emprendimiento e innovación, en diciembre de 2013 en Santiago (Chile). El encuentro, titulado $\mathrm{LAB} 4+$, congregó a funcionarios de alto nivel de los países, expertos, innovadores y emprendedores, para generar las acciones necesarias para construir un ecosistema de innovación y emprendimiento en el área de integración. Así mismo, construyó con los aportes de los asistentes una agenda de iniciativas público privadas que permitan la incubación, crecimiento y posterior realización de emprendimiento o empresas emprendedoras en los cuatro países ${ }^{25}$.

El evento se desarrolló en paneles regionales que comprendían los temas de internacionalización del emprendimiento, financiamiento, educación al servicio de la innovación y el emprendimiento, transferencia de tecnología, superación de brechas y competitividad, y el

25 Alianza del Pacífico (2014) Proyecto Red de Investigación Científica en materia de Cambio Climático. impacto social del emprendimiento y la innovación (Alianza del Pacífico,2013, "LAB4+"). Esta actividad conjunta es una muestra concreta del impulso a la innovación y el emprendimiento donde participa Colombia (Diario Gestión. 2013, "Alianza del Pacifico acuerda fortalecer el emprendimiento e innovación).

\section{Un espacio para el liderazgo}

Adicionalmente, la AP le abre a Colombia una oportunidad de liderazgo ${ }^{26}$ internacional a partir de la persuasión ${ }^{27}$, pues permite construir consensos a favor de sus propios intereses desde los temas en que puede aportar sus capacidades institucionales y experiencia técnica.

En ese sentido, la AP le brinda a Colombia un espacio para desarrollar una diplomacia de potencia media, consolidando relaciones de confianza y consensos con países miembros y observadores hacia la integración y el reconocimiento regional. Ese espacio permitirá a Colombia desarrollar una diplomacia de potencia media ${ }^{28}$, consolidando

26 El liderazgo es un proceso que tiene varias definiciones desde la administración, la ciencia política y las relaciones internacionales. Desde la administración, se concibe como un proceso en el cual se construyen relaciones de influencia hacia la obtención de resultados y/o la construcción de vínculos humanos con unas características, relaciones y conductas estudiables (Pariente, pp. 153189). Desde la política, está asociado a unas habilidades para su ejercicio, a una superioridad en el ejercicio de la influencia entre pares y en la capacidad para focalizar las expectativas del Grupo o de fijar metas (Delgado, 2004, pp. 7-30). Finalmente, para los fines de este artículo se toma la perspectiva de las relaciones internacionales, en la cual se entiende como la influencia de un Estado sobre otros para movilizar el Acuerdo o la acción hacia intereses comunes (Albarracín, pp. 1-4).

27 Según Albarracín, el liderazgo internacional es un proceso que se puede construir desde las capacidades de fuerza, la cooptación con recursos o la persuasión basada en argumentos y propuestas certeras frente a los problemas internacionales. Ese proceso significa para el Estado que lo emprende incurrir en costos mayores en el tipo de liderazgo a construir, se implementa mediante alianzas regionales e implica el reconocimiento de los otros Estados para su realización. (Albarracín,:pp. 1-4).

28 Al respecto, según Evans (2011), «Middle power 
relaciones de confianza e integración con países miembros y observadores hacia el reconocimiento regional. Al respecto, Morales Fajardo y Almonte (2012) reconocen que la AP «(...) no representa el liderazgo mexicano porque, como se ha observado, fue una iniciativa peruana y que, hoy en día, también Colombia imprime su sello en la negociación» (p. 128).

De esta manera, la AP le abre a Colombia la puerta construir un área de integración sin tensiones con otros países o grupos regionales, diversificar sus relaciones temáticas y geográficas, así como la oportunidad para construir un liderazgo sobre la generación de consensos. Todos estos factores hacen de la AP un espacio que fortalece su política exterior.

\section{CONCLUSIÓN}

La Alianza del Pacífico (AP) es un área de integración que ha tenido la atención de la prensa, los centros de pensamiento y diversos autores, los cuales la han estudiado como un actor unitario. No obstante, en la literatura sobre el tema no se analizaba en detalle las ventajas de la Alianza para los Estados participantes como Colombia, y en particular, para su política exterior.

En ese contexto, este artículo demuestra que la AP tiene ventajas para la inserción internacional

diplomacy, is, in short, the kind of diplomacy which can, and should, be practiced by states which are not big or strong enough, either in their own region or the wider world, to impose their policy preferences on anyone else; but who do recognize that that there are international policy tasks which need to be accomplished if the world around them is to be safer, saner, more just and more prosperous [...] and who have sufficient capacity and credibility to be able to advance those tasks." Así mismo, el mismo autor explica más adelante que "The characteristic method of middle power diplomacy is coalition building with "like-minded" countries. It usually also involves "niche diplomacy", which means concentrating resources in specific areas best able to generate returns worth having, rather than trying to cover the fields. de Colombia. Así, se analizaron las ventajas de la AP para Colombia, a través de un enfoque ecléctico que emplea elementos de los estudios sobre la integración regional y del análisis de política exterior.

En este artículo logramos demostrar que la AP genera ventajas para la inserción internacional de Colombia, que están basadas tanto en las características de esa área de integración como en las oportunidades que abre para su política exterior. La Alianza permite desde sus instituciones una agenda definida de integración, la cosecha temprana de las negociaciones, el seguimiento a las cumbres y una presidencia pro témpore eficaz, que hacen de la AP un área de integración con credibilidad. Por otra parte, le ofrece a Colombia un área de integración sin tensiones con otros actores, instrumentos para diversificar sus relaciones con el mundo y una opción de liderazgo regional desde la persuasión.

De esta manera, la AP constituye un área de integración valiosa para la inserción internacional del país hacia el futuro. Una Alianza que le facilita generar beneficios concretos de integración y proyección internacional con su participación.

\section{REFERENCIAS}

Albarracín, J. (2005). Buscando el liderazgo en la región. La política exterior brasilera hacia Sudamérica. Disponible en: http://www. redintercol.org/Albarracin-Juan-Buscandoliderazgo-region.pdf

Alianza del Pacífico. (2013, octubre 24). Científicos de la Alianza del Pacífico debatieron en Chile sobre cambio climático. Consultado enConsultado en www.alianzadelpacifico.net/ científicos-se-paises-de-la-alianza-del-pacificodebatieron-en-chile-sobre-cambio-climatico/ en diciembre 30 de 2013.

Alianza del Pacífico. (2013, julio 17). Con la aceptación de Turquía, Corea del Sur, China 
y Estados Unidos la Alianza del Pacífico suma 20 Países Observadores. Consultado en http://alianzaPacífico.net/con-la-aceptacionde-turquia-corea-del-sur-china-y-estadosunidos-la-alianza-del-Pacífico-suma-20-paisesobservadores/ en julio 24 de 2013.

Alianza del Pacífico. (2013, mayo 14). Países de la Alianza del Pacífico compartirán sedes de sus embajadas. Consultado enConsultado en http://www.viicumbrealianzadelPacífico.com/ noticias/pa\%C3\%ADses-de-la-alianza-delpac\%C3\%ADfico-compartir\%C3\%A1n-sedesde-sus-embajadas en mayo 20 de 2013.

Alianza del Pacifico. (2013). LAB4 +. Consultado en www.alianzadelpacifico.net/lab4\# expositores Consultado el 30 de diciembre de 2013.

Alianza del Pacífico. (2014) "Proyecto Red de Investigación Científica en materia de Cambio Climático", contenido del link sobre Cooperación de la Pagina Web de la Alianza del Pacifico. Consultado en http://alianzapacifico. net/en/cooperacion/ en enero 2 de 2014.

AMEXCID. (2013, octubre 25) Científicos de países de la Alianza del Pacífico debatieron en Chile sobre cambio climático. Consultado enConsultado en www.amexcid.gob.mx/index.php/es/prensa/comunicados/1918-científicos-de-paises-de-la-alianza-del-pacifico-debatieron-en-chile-sobre-cambio-climatico en diciembre 30 de 2013.

Ardila, M., Cardona, D., \& Ramírez, S. (Eds.). (2005) Colombia y su política exterior en el siglo XXI. Bogotá: CEREC - FESCOL.

Ardila, M. (2011) Prioridades e instrumentos de la inserción internacional de Colombia. En Cardona, D (editor). Colombia: Una Política Exterior en Transición. Bogotá: FESCOL.

Ardila, M. (2012). La Cumbre de las Américas. Entre el Nuevo y el Viejo Multilateralismo". Ponencia presentada en Foros Camino a Cartagena, Bogotá .
Banco Interamericano de Desarrollo (2013, Diciembre 5) Primer foro de innovación y emprendimiento de la alianza del pacifico. Disponible en: www.iadb.org/es/temas/comercio/ primer-foro-de-innovacion-y-emprendimiento-de-la-alianza-del-pacicifo,8836.html consultado en diciembre 30 de 2013.

Borda, S., \& Tickner, A. (Eds). (2011). Relaciones internacionales y politica exterior de Colombia. Bogotá: Ediciones Uniandes.

Cardona, D. (editor). (2011). Colombia: Una Politica Exterior en Transición. Bogotá: FESCOL.

Caporaso, J. A. (1992). "International relations theory and multilateralism: the search for foundations" International Organization, 46, 3.

Dade, C., \& Meecham, C. (2013, Julio 11). The Pacific Alliance: an example of lessons learn. Americas - a closer look, paper seriesWashington: CSIS.

Delgado, S. (2004). Sobre el concepto y el estudio del liderazgo político. Una propuesta de síntesis. En Psicología Política, 29, 7-29.

Departamento Nacional de Planeación. (2010). Prosperidad para todos. Bases del Plan Nacional de Desarrollo 2010 - 2014. Bogotá:- DNP.

Diario Gestión. (2013, diciembre 8) Alianza del Pacifico acuerda fortalecer el emprendimiento $e$ innovación. Consultado enConsultado en www.gestion.pe/empresas/alianza-del-pacifico-acuerda-fortalecer-emprendimiento-innovacion-2083070 en diciembre 30 de 2013.

Evans, G. (2011, junio 29). Middle Power Diplomacy. Inaugural Edgardo Boeninger Memorial Lecture en Chile Pacific Foundation, Santiago de Chile.

Farnsworth, E. (2013). The Pacific Alliance a New Center of Gravity in Hemispheric Trade. World Politics Review. Consultado en Disponible en: http://www.worldpoliticsreview.com/ 
articles/12990/the-pacific-alliance-a-new-center-of-gravity-in-hemispheric-trade.

George, S. (2014, marzo 11). The Pacific Pumas. An emerging Model for Emerging Markets. Washington: Bertelsmann Foundation y Global Economic Dynamics.

Ledger, T. (2010). El perfil del multilateralismo latinoamericano. Foreign Affairs Latinoamerica, 10, 3.

Malamud, C. (2012). La Alianza del Pacífico: un revulsivo para la integración regional en América Latina. , Documento ARI 46/2012. Madrid: Real Instituto Elcano.

Morales F, M. E. \& Almonte, L. d. J. (2012) ¿Un nuevo intento a la integración latinoamericana? México y la Alianza del Pacífico. Cuadernos sobre Relaciones Internacionales, Regionalismo y Desarrollo, 7. 14, 11-134.

Palou, J. (1993). El concepto de potencia media. Los casos de España y México". Afers Internacionals, 26, 7-35.

Pariente, J. L. (n.d.). Algunas reflexiones entorno al concepto de liderazgo. En Mendoza, J. (Ed.). Procesos de cambio y desarrollo organizacional, (153 - 189). Disponible en: http://www.researchgate.net/publication/235985767_Algunas_reflexiones_en_torno_al_concepto_dēlliderazḡo

Portales, C. (2012, noviembre). Cumbritis and Prospects for Latin American Regionalism. Consultado en http://aulablog.net/2012/12/20/ cumbritis-and-prospects-for-latin-americanregionalism/ en julio 23 de 2013.

Ramirez, S. (2013). Regionalism: the pacific alliance. Americas Quaterly. Consultado en Consultado en http://www.americasquarterly. org/content/regionalism-pacific-alliance en julio 15 de 2013.
Presidencia de la República de Colombia. (2014, junio). Palabras del Presidente Juan Manuel Santos a México de la Presidencia ProTémpore de la Alianza del Pacífico. Consultado enConsultado en http://wsp.presidencia.gov. co/Prensa/2014/Junio/Páginas/20140620_03Palabras-President... en junio 20 de 2014.

Ramírez, S. (2011). El giro de la política exterior colombiana. Nueva Sociedad, 231, 79 - 95.

Rojas, F. (2010). La Comunidad de Estados Latinoamericanos y Caribeños. Foreign Affairs Latinoamerica, 10, 3.

Revista Eco América. (2013, octubre 30). Científicos de la Alianza del Pacífico debatieron en Chile sobre cambio climático. Consultado enConsultado en www.ecoamerica. cl/noticiaseco/13-noticias/cambio-climatico/1704-científicos-de-paises-de-la-alianza-del-pacifico-debatieron-en-chile-sobre-cambio-climatico.html en diciembre 30 de 2013. Rojas, F. (2012, Octubre 17-19). Ideología y pragmatismo en la participación de Chile en la Alianza del Pacífico. Ponencia presentada en el $X$ Congreso Chileno de Ciencia Política, Santiago de Chile.

San Francisco, A. (2013). La Alianza del Pacífico: un compromiso de futuro. Madrid: Fundación Ciudadanía y Valores.

SELA. (2013, octubre 25). Científicos de países de la Alianza del Pacifico debaten en Chile sobre cambio climático. Consultado enConsultado en http://sursur.sela.org/ listado-de-noticias/2013/10/científicosde-paises-de-la-alianza-del-pacificodebatieron-en-chile-sobre-cambio-climatico en octubre 25 de 2013.

Serbín, A. (2009). América Latina en un mundo multipolar: ¿es la UNASUR la alternativa? Nueva Sociedad, 219,. 


\section{Documentos Oficiales}

Alianza del Pacífico. Acuerdo Marco de la Alianza del Pacífico.

Alianza del Pacífico. Protocolo Adicional al Acuerdo Marco de la Alianza del Pacífico.

Alianza del Pacífico. Protocolo de Cartagena.

Alianza del Pacífico. Declaración de Lima.

Alianza del Pacífico. Declaración de Mérida.

Alianza del Pacífico. Declaración de Paranal.

Alianza del Pacífico. Declaración de Cádiz.

Alianza del Pacífico. Declaración de Cali.

Alianza del Pacífico. Declaración de Cartagena.

Alianza del Pacífico. Declaración de Punta Mita.
Alianza del Pacífico. Lineamientos sobre la participación de los Estados Observadores de la Alianza del Pacífico. Aprobados en la IX Reunión del Grupo de Alto Nivel de la Alianza del Pacífico, los días 15 y 16 de octubre de 2012 en Lima, Perú.

Alianza del Pacífico. Memorando de Entendimiento entre el Gobierno de los Estados Unidos Mexicanos, el Gobierno de la República de Colombia, el Gobierno de la República de Chile y el Gobierno de la República del Perú sobre la plataforma de cooperación del Pacífico. Suscrito en la ciudad de Mérida, Yucatán, México, el 4 de diciembre de 2011.

Alianza del Pacífico. Declaración sobre el proceso de adhesión de Costa Rica a la Alianza del Pacífico. Suscrita por el Presidente de la República de Colombia, Juan Manuel Santos, y la Presidenta de Costa Rica, Laura Chinchilla, en Cartagena el 10 de febrero de 2014. 